\title{
Monophasic Synovial Sarcoma of the Infratemporal Fossa-Case Report and Review of the Literature
}

\author{
Ignacio Mendoza, ${ }^{1}$ Ilson Sepúlveda, ${ }^{2}$ and Geraldine Ayres $^{3}$
}

1. ENT-Head and Neck Surgery Service, General Hospital of Concepcion, University of Concepcion School of Medicine, Concepcion, Chile; 2. Radiology Department; ENT-Head and Neck Surgery Service, General Hospital of Concepcion, Concepcion, Chile; 3. Pathology Department, General Hospital of Concepción, University of Concepcion School of Medicine, Concepcion, Chile

S ynovial sarcoma (SS) represents about 10\% of all soft tissue sarcomas. It is believed that its origin would be found in cells that are related neither to ultrastructural nor to histological features of the synovial tissue. Head and neck is very rarely affected, with the lower extremities being most frequent. Complete resection with or without radiotherapy and chemotherapy is currently considered the best available therapy. This time we present the case of a patient with SS located in the infratemporal fossa, its diagnosis, treatment and evolution. According to our knowledge it is the first reported case in South America.

\section{Keywords}

Synovial sarcoma, head, neck, infratemporal fossa, computed tomography, magnetic resonance

Disclosure: Ignacio Mendoza, Ilson Sepúlveda, and Geraldine Ayres have nothing to disclose in relation to this article. No funding was received in the publication

Compliance with Ethics Guidelines: All procedures were followed in accordance with the responsible committee on human experimentation and with the Helsinki Declaration of 1975 and subsequent revisions, and informed consent was received from the patient involved in this case study.

Authorship: All named authors meet the International Committee of Medical Journal Editors (ICMJE) criteria for authorship of this manuscript, take responsibility for the integrity of the work as a whole, and have given final approval to the version to be published.

open Access: This article is published under the Creative Commons Attribution Noncommercial License, which permits any noncommercial use, distribution, adaptation, and reproduction provided the original author(s) and source are given appropriate credit.

Received: September 19, 2016

Accepted: December 6, 2016

Citation: Oncology \& Hematology Review, 2017;13(1):56-8

Corresponding Author: Ignacio Mendoza, ENTHead and Neck Surgery Service, General Hospital of Concepcion, San Martin Av. 1436; Concepcion, Chile. E: drignaciomendoza@gmail.com
Synovial sarcoma (SS) is a malignant soft tissue tumor, which represents about $10 \%$ of all soft tissue sarcomas. ' It occurs most often in the lower extremities of young men, and is very rare in the head and neck region, with an incidence of about $2-4 \%{ }^{2.3}$ The origin of the SS remains uncertain, although it is believed that its origin would be in undifferentiated stem cells and tissue that are related neither to ultrastructural, nor to histological synovial tissue, being only $<5 \%$ intraarticular. ${ }^{4}$ Typically, it affects patients between 14 and 35 years of age, and its clinical presentation includes the presence of a neck mass, hoarseness, dysphagia, and dyspnea. ${ }^{5}$ When it is presented in the head and neck $(\mathrm{HN})$ it seems to have better prognosis than in the extremities, with five-year survival for head and neck between $47 \%$ and $82 \%{ }^{6}$ The first report in HN was in 1954, ${ }^{7}$ and the most frequent location has been in hypopharynx and lymph nodes. Other locations are reported in parotid gland, paranasal sinuses, larynx, pharynx, and the retropharyngeal space. ${ }^{2.8}$ There are only few reports of SS in infratemporal fossa., ${ }^{4,-13}$ The optimal treatment has not been established, but complete resection with or without radiotherapy and chemotherapy is currently considered the best available therapy. Here is a case of synovial sarcoma located in the left infratemporal fossa. We describe its diagnosis, therapeutic confrontation, and evolution.

\section{Case report}

A 32-year-old man showed at the dental office in March 2014, due to pain in his left mandibular temporomandibular joint with about 3 months of evolution. Bruxism was diagnosed and a plane relaxation for 3 months was prescribed; however, the patient did not experience a good response.

In June 2014 he was referred to a maxillofacial surgeon who confirmed a volume increase in the left zygomatic region (see Figure 1). A computed tomography (CT) scan was performed, revealing a multilobulated large, aggresive, expansive, and destructive heterodense solid mass centered in the left masticatory space. The lesion also involved the oval foramen, ipsilateral infratemporal, and pterygopalatine fossa. There was osteolitic compromise of the mandible, temporomandibular join, pterygoid process, middle cranial base, and sphenoid wing. At its largest diameter, the lesion measured $8 \mathrm{~cm}$ and enhancement was seen following intravenous contrast administration with central hypodense areas suggestive of necrosis. Lymphadenopathy was observed with hypodense center in the II ganglionar level as large as $10 \mathrm{~mm}$ were present (see Figure 2C).

Subsequently, a magnetic resonance imaging (MRI) study was performed revealing in T1 sequence a solid isointense expansive process warping the posterior wall of the maxillary sinuse and 
Figure 1: Clinical presentation of the tumor

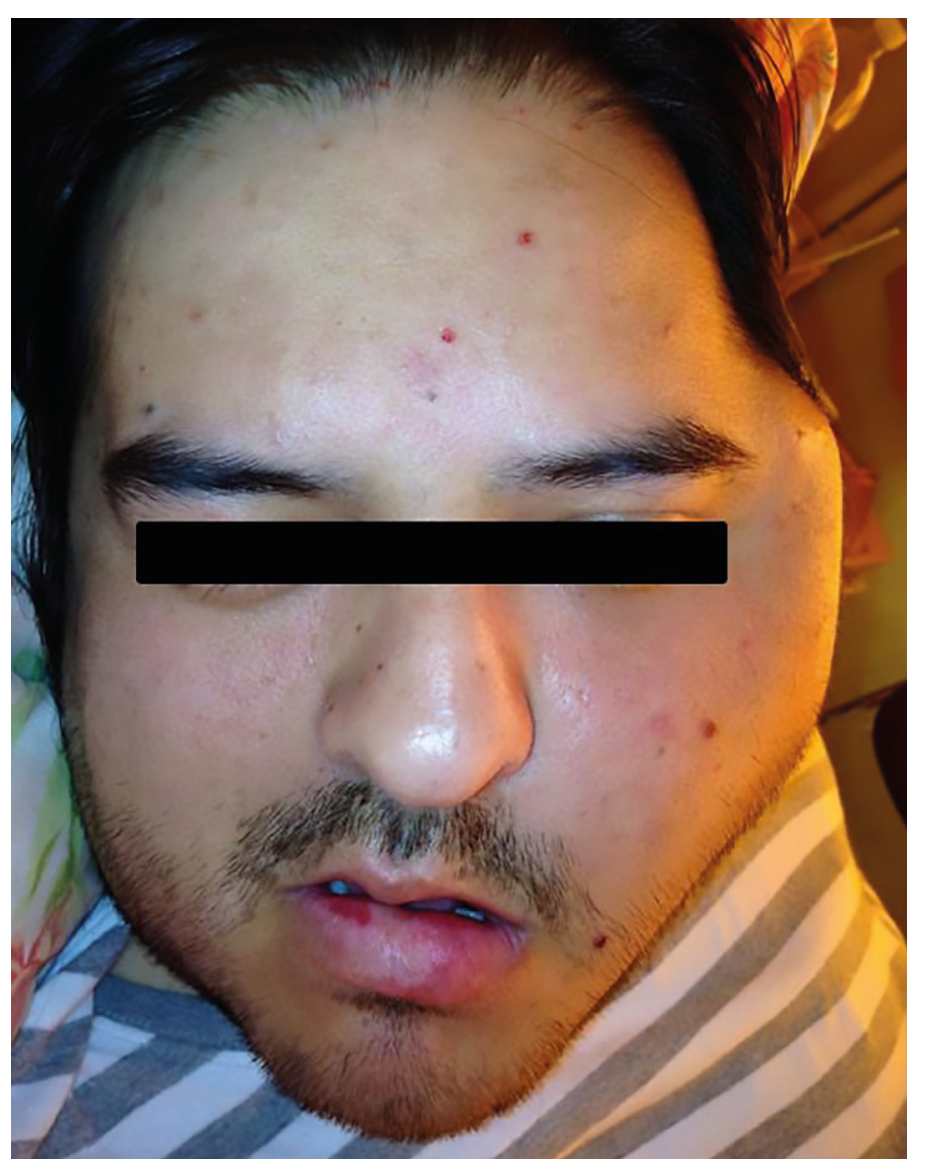

Figure 2: Magnetic resonance imaging

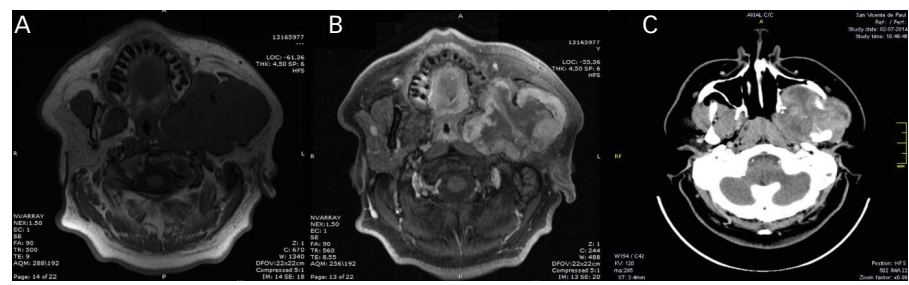

Magnetic Resonance Imaging (MRI). Large solid isointense expansive process warping the posterior wall of the maxillary sinus and parapharyngeal space with moderate enhancement with clear involvement of the internal carotid artery. A: T1 axial view. B: $T 1$ fat sat Gadolinium axial view. (B) T1 axial view after administration of gadolinium contrast. C. Computed Tomography (CT), Soft tissue window post intravenous contrast injection axial view: multilobulated and destructive heterodense solid mass centered in the left masticatory space with osteolitic compromise of the mandible. The intravenous contrast reveals a central hypodense area suggestive of necrosis.

parapharyngeal space (see Figure 2A). An additional MRI T1 sequence following administration of gadolinium contrast material showed moderate enhancement with clear involvement of internal carotid artery (see Figure 2B).

In this context, it was decided to perform incisional biopsy of the lesion the histological analysis was reported as SS (see Figure 3). The study for metastasis was negative. Based on the size of the tumor, the Head and Neck Tumor Board (HNTB) recommended intensity modulated radiation therapy (IMRT) in order to reduce its volume and try posterior surgical resection.
Figure 3: Histopathologic pictures
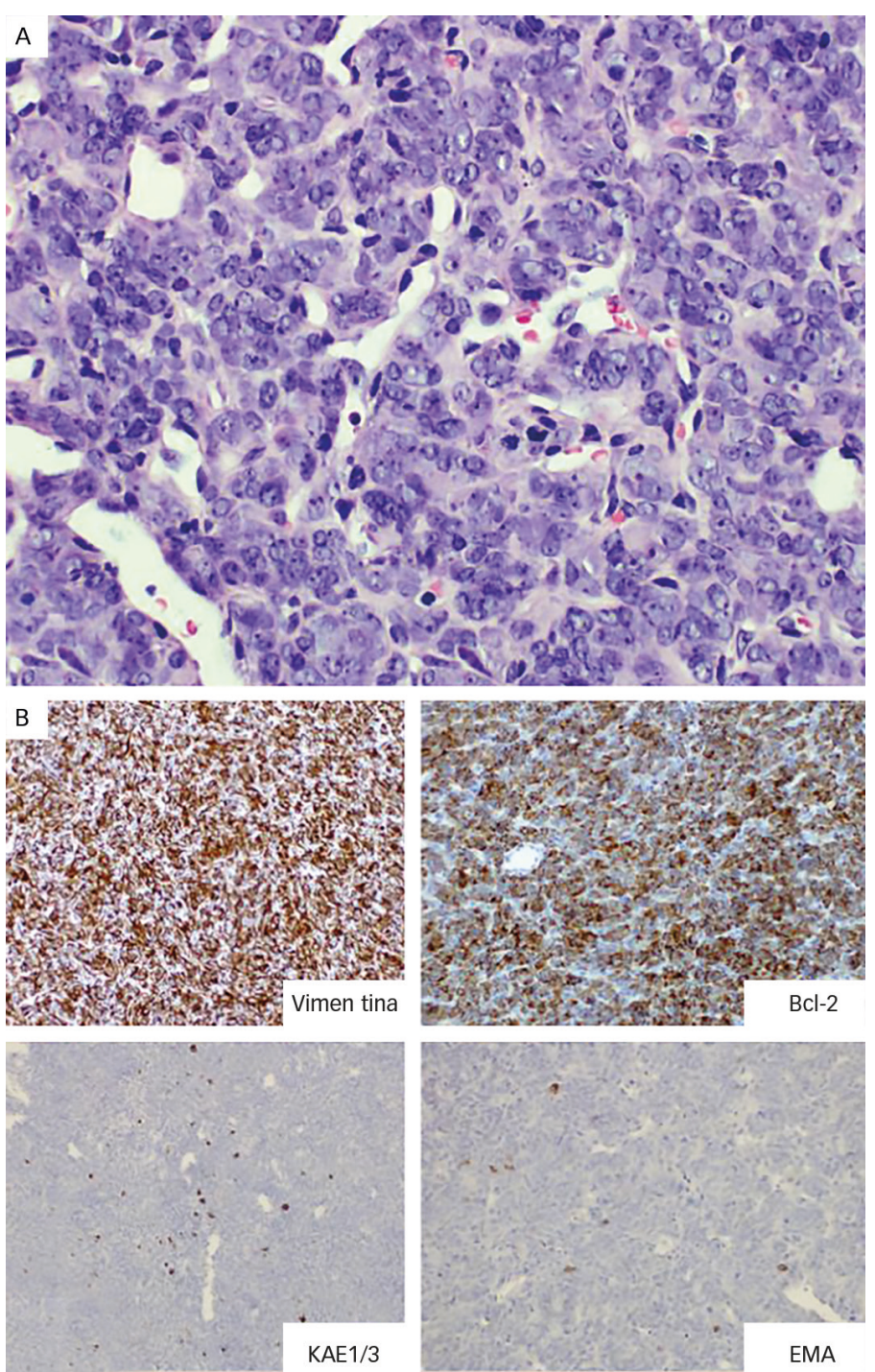

A: hematoxilin and eosin stain; epithelial tumor formed by atypical cells with a hyperchromatic irregular nucleus and prominent nucleoli, arranged in a solid pattern with dilated vessels of hemangiopericitomatoide aspect. B: an immunohistochemical study revealed positive intense diffuse staining for Vimentin and BCL-2, and positive for EMA and focal KAE1/3.

After IMRT, with total dose of $70 \mathrm{~Gy}$, a reduction in tumor mass is achieved; however, this is not enough to raise the total security resection margins, 6 months after treatment was completed, a follow-up computed tomography (CT) exam showed presence of lung nodules suggestive of metastasis. Following additional review by the HNTB, palliative chemotherapy was recommended. He received five cycles of Doxorubicin and ifosfamide with mesna, showing good tolerance, pain decrease and stabilization of pulmonary nodules. Upon finishing this article, 2 years after diagnosis, the patient remains in palliative care, with local complications and deterioration of his performance status.

\section{Discussion}

An epithelial form with columnar cells and other non-epithelial cells with fusiform are observed: SS in two different histological forms. The most common SS showing two types of shapes is known as biphasic. Tumors with only epithelial components are rare, and are called monophasic. 
Differentiation of the latter type with other types of neoplasias is difficult. ${ }^{4}$ The variety of our case corresponded to a monophasic Ss; we performed the diagnosis through intense diffuse positive staining to vimentin and $\mathrm{BCl}-2$, and the positive focal dialing EMA and KAE1/3, which is typical of these tumors.

The current treatment of the SS is based on surgical resection with safety margins, with or without chemoradiotherapy. In HN, complete resection with adequate margins is difficult, and impossible in many cases, given the complex anatomy; however, it generates better results in terms of recurrence and survival. Regarding chemoradiotherapy, several strategies have been reported, with different results.

Some studies reported that postoperative dose radiotherapy of 50-65 Gy in patients with clear safety margins or microscopic residual tumor was effective in reducing local recurrence, but not survival, ${ }^{15-17}$ nor the chemotherapy before or after surgery in two different research works. ${ }^{17,18}$ Therefore, surgical resection with safe margins is most important; chemoradiotherapy effectiveness remains uncertain.

Distant metastases occur in up to $50-70 \%$ of cases, and are frequent in cervical lymph nodes by $10-20 \%$. Overall, it is a poor prognosis, with a five- year survival rate of $25-62 \%$ and $10-30 \%$ for 10 years. ${ }^{1}$ Predictors of worse prognosis for SS have been proposed, such as diameter $>5 \mathrm{~cm}$, biphasic type, primary site based skull, bone involvement, and positive margins after surgical resection. 8,15

The causes of death in SS are recurrences and distant metastases (lungs after cervical lymph nodes). Although local control is difficult, it has been improved with the development of new surgical techniques. In our patient, IMRT treatment was decided due to the size of the tumor, and virtually no possibility of surgical resection with negative margins.

According to our knowledge it is the first reported case in South America. We present the limits of treatment of an infratemporal fossa SS. A more effective therapeutic confrontation is required, especially for tumors that cannot be completely resected.

\section{Conclusions}

According to our knowledge, this is the first reported case of an infratemporal fossa SS in South America. We present the limits of treatment of an infratemporal fossa SS. A more effective therapeutic confrontation is required, especially for tumors that cannot be completely resected. $\square$
1. Harb WJ, Luna MA, Patel SR, et al., Survival in patients with synovial sarcoma of the head and neck: association with tumo location, size, and extension, Head Neck, 2007:29:731-40.

2. Al-Daraji W, Lasota J, Foss R, Miettinen M, Synovial Sarcoma Involving the Head. Analysis of 36 Cases With Predilection to the Parotid and Temporal Regions, Am I Surg Pathol to the Parotid and

3. Ferrari A, Gronchi A, Casanova M, et al., Synovial sarcoma: A retrospective analysis of 271 patients of all ages treated at a single institution, Cancer, 2004;101:627-34.

4. Fisher C, De Bruijn DR, Geurts van Kessel A, Synovial sarcoma. Fletcher CDM, Unni KK, Mertens F, editors. World Health Organization classification of tumours. Pathology and genetics of soft tissue and bone, Lyon: IARC, 2002; pp 200-4.

5. Lai V, Farrag TY, Cao D, et al., Synovial sarcoma of the infratemporal fossa, Am J Otolarygnol Head Neck Med surg, 2007;28:444-7.

6. Kartha SS, Bumpous JM, Synovial cell sarcoma: diagnosis, treatment, and outcomes, Laryngoscope, 2002;112:1979-82
7. Jernstrom P, Synovial sarcoma of the pharynx, Am I Clin Pathol, 1954;24:957.

8. Ji T, Ma CY OW A, et al., Synovial sarcoma involving skull base - A retrospective analysis of diagnosis and treatment of 21 cases in one institution, Oral Oncology, 2011; 47:671-6.

9. Tamarit JM, Estrems P, Estellés E, Dalmau J, Sarcoma sinovial de fosa infratemporal, Acta Otorrinolaringol ESP, 2010;61:389-91.

10. Nomura F, Kishimoto $S$, Synovial sarcoma of the temporomandibular joint and infratemporal fossa, Auris Nasus temporomandibular joint

11. Dhawan A, Shenoy AM, Chavan P, et al., Synovial Sarcoma of the Infratemporal Fossa With Extension Into the Oral Cavity-A Rare Presentation and Literature Review, J Oral Maxillofac Surg 2012;70:2923-9

12. Aslan H, Başoğlu M, Karaca N, Synovial sarcoma of th infratemporal fossa with intracranial extension, Kulak Burun Bogaz Intis Derg, 2012;22:348-53

13. Wushou A, Zhao $Y$, Shao $Z$, Synovial sarcoma of the infratempora fossa: A case report, Oncology Letters, 2014;8:2165-70.
14. Van de Rijn M, Barr FG, Xiong QB, et al., Poorly differentiated synovial sarcoma: an analysis of clinical, pathologic, and moSynovial sarcoma: an analysis of clinical, pathologic, and mo-

15. Lee N, Shin E, Treatment outcomes for patients with synovial sarcoma of the head and neck, Expert Rev Anticancer Ther 2008;8:371-3.

16. Andrassy RJ, Okcu MF, Despa S, Raney RB, Synovial sarcoma in children: surgical lessons from a single institution and review of the literature, I Am Col Surg, 2001;192:305-13.

17. Italiano A, Penel N, Robin YM, et al., Neoadjuvant chemotherapy does not improve outcome in resected primary Sy- novial sarcoma: a study of the French Sarcoma Group, Ann Onco 2009;20:425-30.

8. Pappo AS, Devidas M, Jenkins J, et al., Phase II trial of neoadjuvant vincristine, ifosfamide, and doxorubicin with granulocyte colony- stimulating factor support in children and adolescents with advanced-stage non rhabdomyo sarcomatous soft tissue sarcomas: a Pediatric Oncology Group Study, J Clin Oncol, 2005;23:4031-8. 